\title{
Integrated Plan for LArTPC neutrino detectors in the US
}

\author{
Prepared for the FNAL Director's Review of LArTPC R\&D Planning \\ LArTPC Planning Group, B. Baller and B. Fleming, Editors \\ November 18, 2009
}

\section{Executive Summary}

We present an integrated R\&D plan aimed at demonstrating the ability to build a very large Liquid Argon Time Projection Chamber (LArTPC), on a scale suitable for use as a Far Detector for the LBNE neutrino oscillation experiment. This plan adopts current LArTPC R\&D-related activities and proposes new ones to address questions that go beyond those being answered by the current efforts. We have employed a risk evaluation strategy to identify questions that can be answered (or risks that can be mitigated) through one or more R\&D steps.

In summary form, the plan consists of the following pre-existing components:

- The Materials Test Stand program, now in operation at Fermilab, addressing questions pertaining to maintenance of argon purity

- Exisiting electronics test stands at FNAL and BNL

- The Liquid Argon Purity Demonstrator (LAPD) now being assembled at Fermilab

- The ArgoNeuT prototype LArTPC, now running in the NuMI beam

- The MicroBooNE experiment, proposed as a physics experiment that will advance our understanding of the LArTPC technology, now completing its conceptual design phase.

- A software development effort that is well integrated across present and planned LArTPC detectors.

We are proposing to add to these efforts the following:

- A membrane cryostat mechanical prototype to evaluate and gain expertise with this technology.

- An installation and integration prototype, to understand issues pertaining to detector assembly, particularly in an underground environment.

- $\mathrm{A} \sim 5 \%$ scale electronics systems test to understand system-wide issues as well as individual component reliability.

- A calibration test stand that would consist of a small TPC to be exposed to a test beam for calibration studies, relevant for evaluation of physics sensitivities.

We have developed a timeline and milestones for achieving these goals as discussed in Section 4. The proposed activities necessary for the final design of LAr20 are complete by CD3 in 2014. 
Integrated Plan/page 2

\section{Introduction}

\subsection{LBNE and LAr Science and Project Goals}

Experimental results from the last decade have revolutionized neutrino physics. Most notably, the first conclusive evidence that neutrinos oscillate and have mass was observed only about 10 years ago. While this seminal discovery has answered many questions about the neutrino, it has opened even more. Particularly interesting is the question of CP Violation in the neutrino sector. Do neutrinos and anti-neutrinos oscillate at the same rate? The answer to this question could suggest neutrinos are the key to our matter dominated universe.

Long baseline neutrino oscillation searches are proposed as a way to look for these differences. Moreover, these experiments are the only ones that can simultaneously measure $\theta_{13}$, the last unknown mixing angle in the $3 \times 3$ neutrino mixing matrix, the $\mathrm{CP}$ violating phase, $\delta$, and the neutrino mass hierarchy.

The US particle physics community is developing ideas for the next generation of long baseline oscillation experiments beyond $\mathrm{NO} \nu \mathrm{A}$. To be sensitive to the physics goals, the experiments to be undertaken will need baselines in the range of 250 to $1300 \mathrm{~km}$ and neutrinos with energies in the range of 0.5 to $5 \mathrm{GeV}$. Since we know the oscillation probabilities that depend sensitively on $\theta_{13}$ are small, intense neutrino beams and large detectors are essential. As suggested in the recent report from the Particle Physics Project Prioritization Panel (P5), the most promising path would involve a new intense neutrino beam generated at Fermilab and directed to a far site, for example capitalizing on the proposed NSF Deep Underground Science and Engineering Laboratory (DUSEL). Systems of detectors with target masses in the range of tens to hundreds of kilotons will need to be constructed at the far site. The US Department of Energy is presently in the process of establishing the mission need (CD-0) for a experimental program of this scope, and a project organization, currently denoted as LBNE, has been set up, with a number of national laboratories (BNL, Fermilab, LANL and LBNL) holding project management responsibilities.

Liquid Argon Time Projection Chambers (LArTPCs) are ideal detectors for this program: their signal efficiency and background rejection capabilities are extraordinary, and they appear scalable to large volumes. It is the combination of spatial information and $d E / d x$ of tracks that makes particle identification in the LArTPC very powerful. For these reasons, LArTPCs are the ideal detectors for $\nu_{e}$ appearance signatures like those sought by MicroBooNE, an approved Fermilab experiment that will deploy a 170-ton LArTPC on site, as well as by near-term and future long baseline experiments. In these experiments, high efficiency for $\nu_{e}$ signal events is essential in light of the small oscillation probabilities, and backgrounds from misidentified $\nu_{\mu}$ interactions, including processes involving prompt $\pi^{0}$ production and others that produce $\gamma \mathrm{s}$, must be rejected. Hence, the LArTPC is a natural choice for a Far Detector for the LBNE project.

The ICARUS experiment has pioneered this technology over the last 25 years culminating in successful commissioning of a T300 module in 2001. However, these detectors have not yet been built at the scale necessary for the next generation of long baseline searches.

Within the LBNE project, proponents of the LArTPC approach are in the process of developing a conceptual design for an initial detector module to be installed at the far site, of mass 20 kilotons. Nominally, additional modules would be installed subsequently to reach a total active mass of 50 to $100 \mathrm{kt}$. The initial 20-kt module is referred to here as LAr20. One concept, shown in Figure 1, has large rectangular TPC modules stacked inside a large cryostat. This so-called "membrane style" cryostat is externally supported by the cavern walls, and represents a technology now commonly in use for liquefied natural gas (LNG) transport ships and underground storage 
Shown - 48 dual TPC detector basic units $5 \mathrm{~m} \times 5 \mathrm{~m} \times 40 \mathrm{~m}=12,000 \mathrm{~m}^{3}, 16.8 \mathrm{kt}$ active volume $\mathrm{LAr}$ $22.5 \mathrm{~m} \times 17 \mathrm{~m} \times 42.5 \mathrm{~m}=16,256 \mathrm{~m}^{3}, 22.76 \mathrm{kt}$ Total LAr volume Active $/$ Total Volumes $=74 \%$

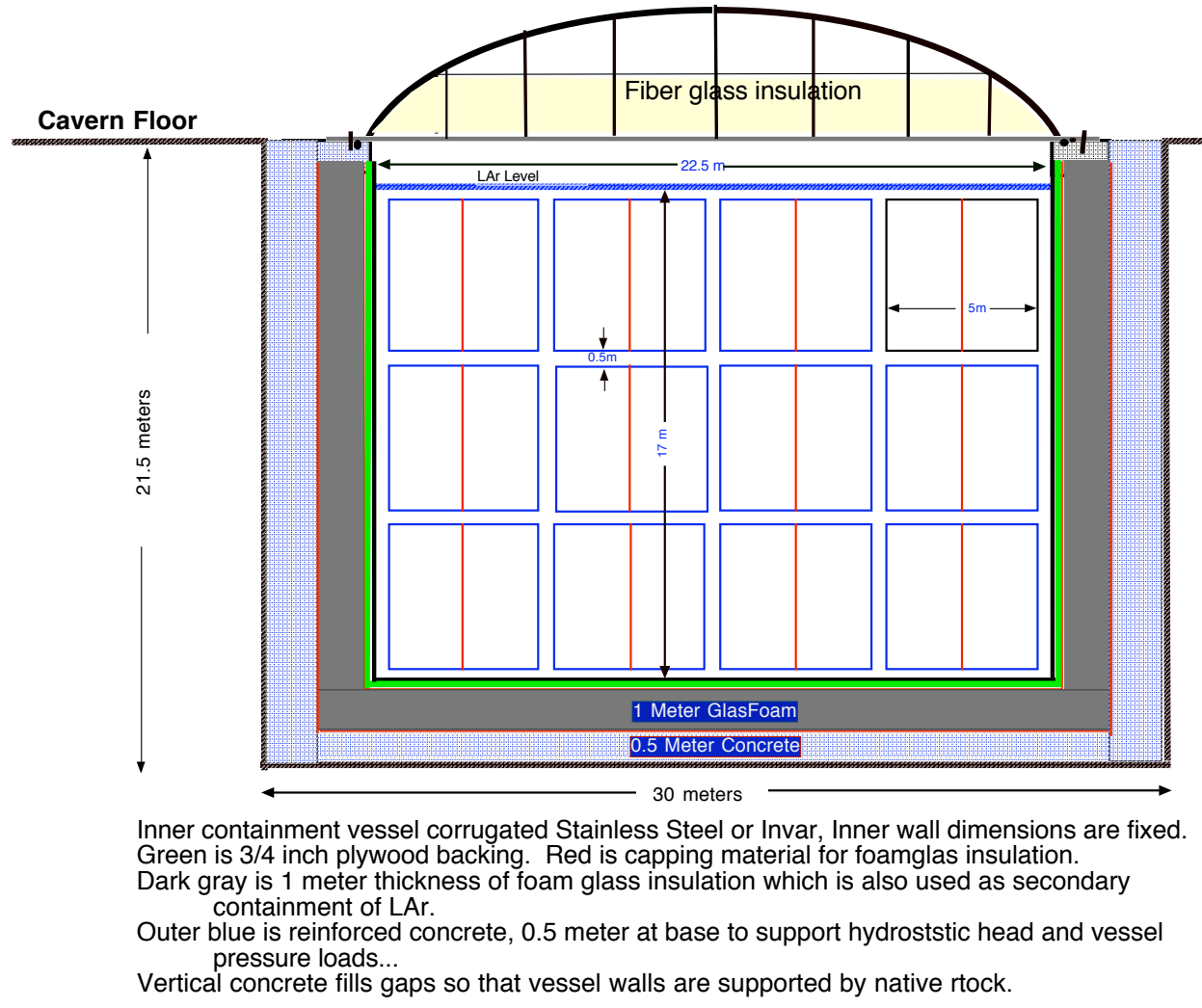

Native Rock Supports Vessel Walls

Figure 1: A schematic of the membrane cryostat conceptual design for LAr20.

facilities. The cryostat would not be evacuable, and would be insulated mainly by a layer of foam glass surrounding the membrane to maintain an external heat load of $\sim 5 \mathrm{~W} / \mathrm{m}^{2}$. It is proposed that readout of signals from the TPC would be achieved with CMOS-based front-end electronics immersed in the liquid argon.

\subsection{Scope and Organization of this Report}

In this document we present our vision for a coherent US LArTPC R\&D program in the context of the LBNE project. Specifically, this plan addresses the questions that need to be answered in order to demonstrate that the LAr20 detector can be constructed, given what we know now. This plan assumes that projects already underway (such as the LAPD purity demonstrator and the construction and operation of the MicroBooNE detector) will be completed successfully, and we consider these projects as elements of the integrated R\&D plan we are proposing.

Recognizing the need to proceed rapidly in light of the current LBNE Project development timeframe, it is important to note the distinction between the R\&D plan and the actual LAr20 
design process, which is proceeding in parallel. In the plan presented here, we are not addressing LAr20 design details. Rather we are addressing the assumptions that form the underpinnings for the detector design process. For example, consideration of a membrane cryostat follows from the notion that the costs and challenges of constructing a 20-kt vessel that can be evacuated may be significant; but can one demonstrate that the required level of argon purity can be achieved without first evacuating the vessel? Do we understand that the membrane cryostat technology, pioneered for the storage and transportation of liquefied natural gas (LNG), can be successfully adapted to the needs of an instrumented volume of liquid argon? Similarly, LAr20 will require the installation of order $10^{6}$ channels of electronics into a device that will not easily be accessible. Can we demonstrate that the electronics for such a detector can operate without malfunction for an extended period under these conditions? To identify the questions to be addressed by the R\&D program, we have employed a risk evaluation methodology that will be described in detail in this report.

The document is organized as follows:

- Outline of the challenges for construction and operation of LAr20

- Outline of the integrated plan to address these challenges

- The present program through MicroBooNE

- Analysis of the risks and mitigations beyond the present program

- Summary

\section{Outline of the Challenges for LAr20}

The main challenges in scaling LArTPCs include issues related to hardware R\&D, physics $\mathrm{R} \& \mathrm{D}$, and costing. These challenges are listed here and discussed further in Section 5.

- Hardware R\&D

- Argon Purity: Achieve and maintain purity in an un-evacuable vessel.

- Electronics: Develop cold, low-noise electronics with channel multiplexing in the LAr.

- Active detector systems (TPC): Design TPC modules and light collection systems.

- Cryogenics: Develop cryogenics systems for operation deep underground.

- Underground Issues: Address issues related to siting of a detector deep underground.

- Physics R\&D

- Analysis tools: Develop tools to simulate and reconstruct neutrino interactions

- Surface operation of a physics experiment: Test feasibility to reconstruct data in a detector exposed to cosmic ray backgrounds on the surface.

- Physics results: Produce publishable physics results with a large experiment 
Integrated Plan/page 5

\section{The Integrated Plan}

The integrated plan presented here addresses the main challenges for LAr20 with the focused R\&D efforts necessary for LAr20, building on the existing program. In this section, the key ingredients of the integrated plan are described. How the present program through MicroBooNE addresses these ingredients is presented, and the steps beyond MicroBooNE to get to LAr20 are developed in subsequent sections. Justification for these steps follows in Section 5: Challenges and Mitigations for LAr20 beyond MicroBooNE.

\subsection{Ingredients for the Integrated Plan}

Below are the key ingredients of this integrated plan. The challenges and the proposed steps to address these are described in more detail in the next sections.

- Integrated Hardware Development: The combination of test stands and experiments in this Integrated Plan address the R\&D questions on a timescale necessary to plan for LAr20. The test stands and experiments are as follows:

- Materials Test Stand: Existing facility to test materials for impurities and to test filtration techniques, relevant to argon purity

- Electronics Test Stand: Existing test stands at FNAL and BNL to develop cold electronics, relevant to electronics development

- Liquid Argon Purity Demonstrator: 30 ton tank built in industry to test gaseous argon purge of un-evacuable vessel, relevant to argon purity

- ArgoNeuT: 0.4 ton LArTPC running in the NuMI neutrino beam to collect 10-20k low energy neutrino interactions, relevant to underground issues and physics $R \& D$

- MicroBooNE: 100 ton active volume LArTPC experiment to run in the on-axis Booster Neutrino Beam, relevant to argon purity, electronics, active detectors, and cryogenics

- Calibration test: Small TPC to be exposed to a test beam for calibration studies, relevant to physics R\&D

- Membrane cryostat mechanical prototype: Small ( 20ton) prototype of the membrane style cryostat for potential leaks and contamination of LAr, relevant to argon purity

- Installation and integration prototype: Test of full integration for construction of LArTPC deep underground, relevant to active detector systems and underground issues

- Electronics $\sim 5 \%$ test: Test and possible re-use of $\sim 5 \%$ of the electronics to be used in LAr20, relevant for electronics

- Integrated Software Development: The Liquid Argon Software group (LArSoft) is a coordinated effort for software development for LArTPCs. The "LArsoft" meetings, are attended by ArgoNeuT, MicroBooNE, and LAr20 collaborators. The analysis tools for simulation, reconstruction, and analysis are shared amongst these tests and experiments taking neutrino data. This allows for easy coordination and ensures no duplication of effort.

\subsection{The present program through MicroBooNE}

There is already a growing program in the US underway as shown in Figures 4 and 3 to address the challenges for LAr20. A description of the present program and the R\&D which will be accomplished is below. 


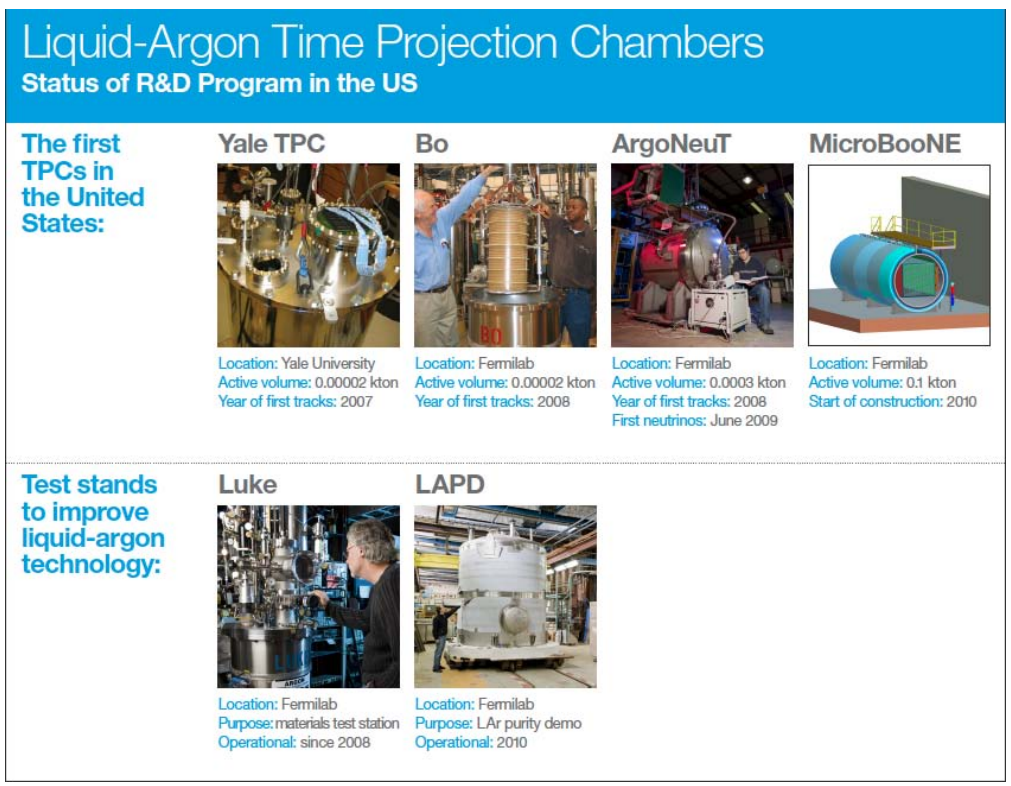

Figure 2: Present program for LArTPC development in the United States

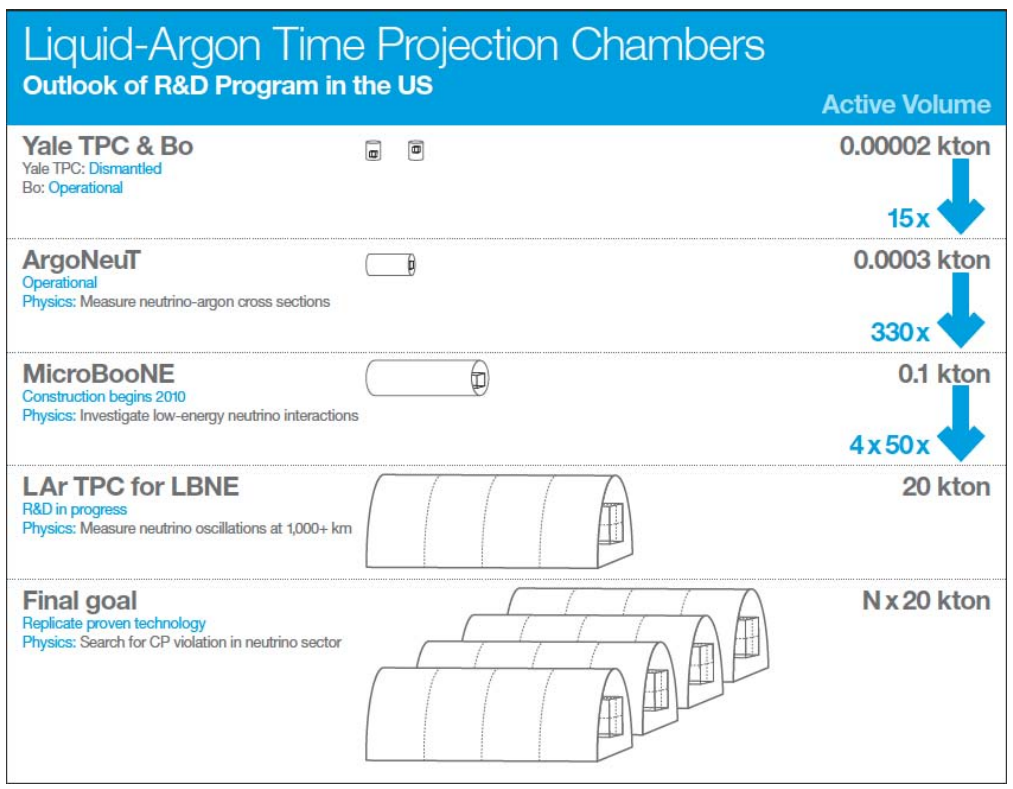

Figure 3: Scaling of LArTPC prototypes and experiments up to LAr20 and beyond 


\subsubsection{Test stands}

The Materials Test Stand, dubbed "Luke", is a facility at Fermilab where different materials can be tested in argon to determine their impact on argon purity. Different filtration materials are also tested for their ability to achieve and maintain pure argon. The facility is both a place to study argon purity and test all materials that go into LArTPCs for the present and future program.

The electronics test stands at Fermilab and Brookhaven can test different electronics designs from the front end boards through readout and DAQ.

\subsubsection{ArgoNeuT}

The Argon Neutrino Test is a 175 liter LArTPC presently running in the NuMI neutrino beam (see http://t962.fnal.gov). The $0.5 \mathrm{~m} \times 0.5 \mathrm{~m} \times 1 \mathrm{~m}$ TPC is housed in an evacuable cryostat and is on beam center directly upstream of the MINOS near detector which is used as a muon catcher for the neutrino interactions. ArgoNeuT began collecting data using the NuMI anti-muon neutrino beam in October 2009 and will run until March 1, 2010. Beyond this run, ArgoNeuT may move to another location in the NuMI near hall or to another beam on site. ArgoNeuT's $\sim 10 \mathrm{k}$ events will allow for development of analysis tools, and first measurements of neutrino cross sections on argon as well as data to better understand signal and background identification for LAr20.

\subsubsection{LAPD}

The Liquid Argon Purity Demonstrator (LAPD) is a test stand whose goal is to demonstrate that the required electron lifetime can be achieved in a cryostat without evacuation. The test stand consists of a 30 ton capacity cryostat, the associate liquid argon purification plant, and the instrumentation necessary to measure electron lifetime in the liquid argon, the temperature gradients in the bulk volume and the flow of the liquid in the bulk. The vessel was built by a standard industrial fabrication company and is currently located in PC4 on the Fermilab site.

The test stand has two phases which will begin in the summer of 2010 . The first phase is a proof of principal of the "Argon Piston" technique for removing oxygen and water from the vessel. In this technique, gaseous argon will be pumped into the bottom of the tank to push the lighter atmosphere out through valves on the top of the vessel. The gaseous argon will cycle through the filter system until the oxygen contamination is less than 50 parts per million. At that point liquid argon will be introduced in the system and cycled through the filters until the contamination is $<$ 100 parts per trillion. For the second phase, the liquid argon will be drained from the vessel and the components of a TPC, including structural elements and cables, will be placed in the vessel. The procedure from the first phase will be repeated to show that the technique works in the presence of a TPC.

After completion of these initial phases, the LAPD may have an active TPC installed in it in order to provide a test bed for cold electronics, light collection devices and refinements to the purification technique.

\subsubsection{MicroBooNE}

The MicroBooNE experiment is a 100 ton active volume LArTPC in the design and prototyping phase. It has both a physics program and LArTPC Development goals. The physics program's goals are:

- Search for and resolve the low energy excess observed by the MiniBooNE experiment 
- Perform a suite of low energy, precision cross section measurements

MicroBooNE's development goals are:

- Demonstrate photon-electron identification

- Develop cold electronics

- Implement cold electronics in gaseous argon

- Develop and test cold electronics in LAr in MicroBooNE

- Test of gaseous argon purge to achieve good purity without evacuation, in a fully instrumented physics experiment

- Refine sensitivity estimates for LBNE/LAr20 program

- Test ability to run on the surface for non $\nu$-beam physics

- Develop tools for analysis

- Develop cost scaling model for larger detectors

MicroBooNE received stage 1 approval from the Fermilab director in 2008, partial funding through an NSF MRI in 2008 and an NSF proposal in 2009 and DOE CD-0 Mission need in 2009. MicroBooNE's DOE CD-1 review date is set for March 2010. Design and prototyping are ongoing. MicroBooNE's scheduled CD-4 is in 2013.

\subsection{The Program Beyond MicroBooNE}

The challenges and mitigations for LAr20 beyond MicroBooNE were determined in the context of developing a risk matrix for LAr20 given what we will learn through MicroBooNE. A consequence of this risk analysis is the proposed new R\&D activities listed below. The justification for this proposal can be found in Section 5 .

\subsubsection{0t Cryostat Prototype}

In the case that the reference design is a membrane cryostat, a 30t operable membrane cryostat prototype will be constructed. The purpose of this activity is:

- To gain experience in construction and operation of the vessel

- To achieve and maintain purity in a membrane vessel

These goals can be achieved with this relatively "small" 30t vessel. It is possible that the 30t LAPD vessel and cryogenic system may be re-used for this activity.

\subsubsection{Installation Mock-up}

Underground assembly and installation of a TPC module inside a cryostat will be challenging. The Installation mock-up will allow for assembly and installation of one full-scale TPC module in a mock-up of the cryostat. This will mitigate the risks associated both with underground installation and with integration of the different systems in the cryostat. The mock-up will simulate underground conditions and allow for time and motion studies. If possible, the full-scale TPC module will be re-used for LAr20. 


\subsubsection{Electronics Test}

A series of small scale electronics tests will be performed while the electronics design is developed. A test of $\sim 5 \%$ of the electronics is envisioned to ensure the optimal operation of and long term reliability of the cold electronics. The vessel used for LAPD and the 30t cryostat prototype may be available for this cold electronics test.

\subsubsection{Calibration Test}

A calibration test of detector response to electrons and hadrons would benefit the data analysis for LAr20. The MTest facility at Fermilab provides electron and hadron beams down to $500 \mathrm{MeV}$ and up to many $\mathrm{GeV}$, the relevant energy range for LBNE. This test should have a TPC as much like the final design for LAr20 and does not need to be completed before LAr20 will run, at CD4.

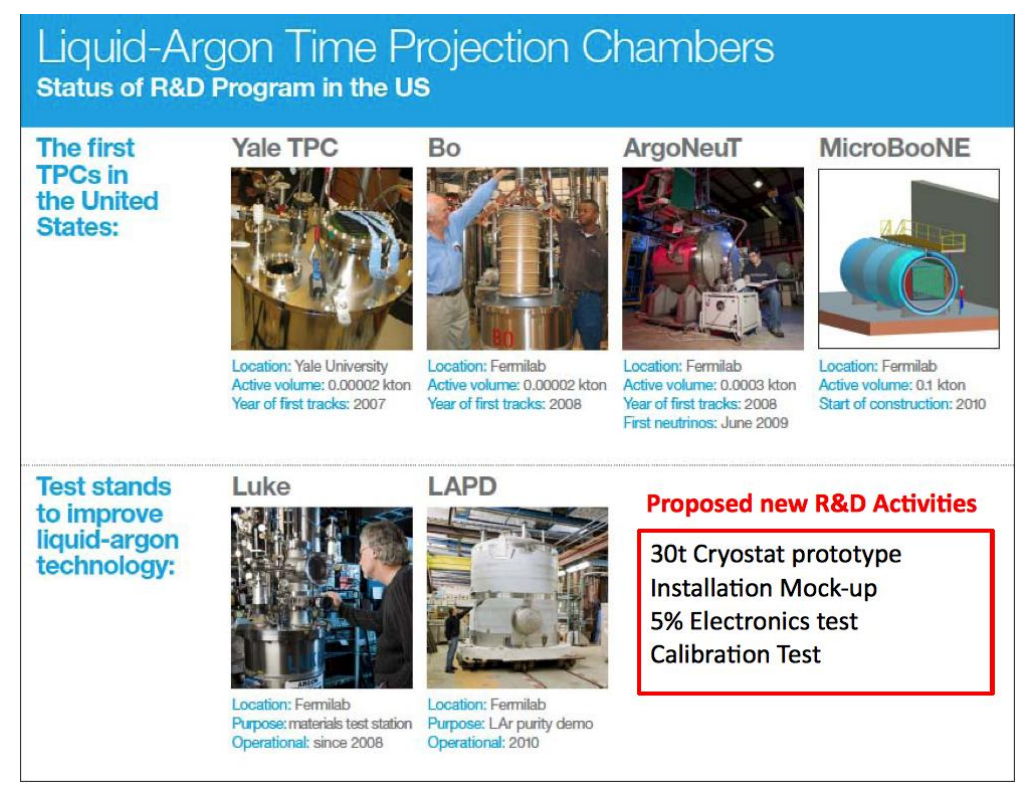

Figure 4: Present plan and proposed activities beyond this.

Milestones for the Integrated Plan have been developed and are shown in Table 4.3.4 below. 
Integrated Plan/page 10

\begin{tabular}{|l||l|l|l|l|l|l|l|l|}
\hline \multicolumn{7}{|c|}{ Integrated Plan Milestones } \\
\hline
\end{tabular}

Table 1: Milestones in the Integrated plan needed to achieve CD3 by 2014 and CD4 by 2018. 


\section{Challenges and Mitigations for LAr20 beyond MicroBooNE}

To date, the liquid argon R\&D plan has included the initiatives decribed in Section 4.2 . Over the past three months we have a conducted a risk analysis to identify areas of weakness in the plan and to propose new initiatives to mitigate risks that have not been considered. The risk analysis methodology here is an adaptation of a standard technique used for project management, specifically the NSLS-II project. The main elements are risk identification, risk classification, and the presentation of risk mitigation action items (e.g. R\&D activities).

This analysis was conducted by a group of people who have relevant experience in U.S. and European R\&D activities: Bruce Baller, Flavio Cavanna (LAquila), Bonnie Fleming (Yale), Cat James, Ornella Palamara (Gran Sasso), Stephen Pordes, Rob Plunkett, Gina Rameika, Brian Rebel and Jon Urheim (Indiana).

\subsection{Risk Identification}

We identified the residual risks that will remain after the currently envisioned R\&D plan is completed by proposing a scenario where all of the currently planned R\&D activities are successful:

- ArgoNeut is currently taking data in the NuMI neutrino beam. Current indications are that it will achieve its R\&D and physics goals.

- The LArSoft group has completed work on a detailed Monte Carlo simulation and has begun developing reconstruction software. At the current pace of development, full 3D track and electromagnetic shower reconstruction will allow detailed physics studies to begin in late 2010 .

- First results from LAPD are expected in the summer of 2010. For the risk analysis we assume that LAPD will demonstrate the ability to achieve long electron lifetime without first evacuating the cryostat.

- MicroBooNE is scheduled to begin operation in 2013. For the risk analysis we assume that MicroBooNE will provide a second demonstration of the ability to achieve long electron lifetime in a fully functioning TPC without first evacuating the cryostat. We furthermore assume that by this time analysis tools will have been developed to reconstruct neutrino events and MicroBooNE physics results are imminent.

The set of residual risks (See Figure 7) was presented to the LBNE collaboration in October and to the LAr20 working group in November to ensure completeness. We consider the November 23 Directors Review of this R\&D plan to be a further quality assurance check.

\subsection{Risk Classification}

Each risk was discussed by the group to reach a consensus determination of the consequence if the event were to occur and likelihood of its occurrence. We used the guidelines shown in Figure 5 to categorize the consequence and the likelihood. The product of the consequence and likelihood are used to define the risk (Low, Med, and High) using the matrix shown in Figure6. This procedure is not quantitative despite its appearance. The main value lies in its utility as a tool to reach a group consensus on the relative level of risk. It therefore highlights important issues that are addressed in the next phase. 


\begin{tabular}{|l|c|l|}
\hline Consequence & Ranking & \multicolumn{1}{|c|}{ Threshold } \\
\hline Very High & 3 & Significant delay ( $>1$ year), cost impact $>\$ 10 M$ \\
\hline Moderate & 2 & Moderate delay ( $>3$ months), cost $>\$ 1 M$ \\
\hline & 1 & Minor delay (<3months), cost impact $<\$ 1 M$ \\
\hline
\end{tabular}

\begin{tabular}{|l|c|l|}
\hline $\begin{array}{l}\text { Likelihood of } \\
\text { occurrence }\end{array}$ & Ranking & Threshold \\
\hline Very likely & 3 & $>90 \%$ probability \\
\hline Likely & 2 & $50 \%-90 \%$ probable \\
\hline Unlikely & 1 & $1 \%-50 \%$ probable \\
\hline Very Unlikely & 0 & $<1 \%$ probable \\
\hline
\end{tabular}

Figure 5: Consequence and likelihood rankings and thresholds

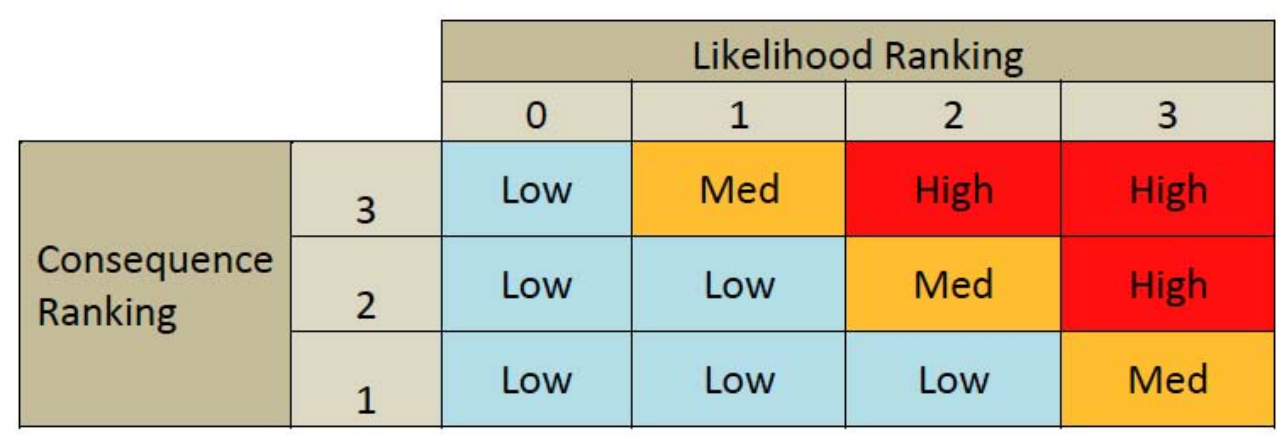

Figure 6: Risk determination from consequence and likelihood rankings 


\begin{tabular}{|c|c|c|c|c|c|c|c|}
\hline Category & Post MicroBooNE Potential Problem & $\begin{array}{l}\text { Conse } \\
\text { quence }\end{array}$ & Likelihood & Risk & Mitigation & $\begin{array}{c}\text { Mitigated } \\
\text { Likelihood } \\
\end{array}$ & $\begin{array}{c}\text { Mitigated } \\
\text { Risk }\end{array}$ \\
\hline Argon Purity & $\begin{array}{l}\text { Achievement of adequate lifetime requires } \\
\text { evacuation. (MicroBooNE and/or LAPD were not } \\
\text { successful) }\end{array}$ & 3 & 1 & Med & $\begin{array}{l}\text { Keep modular cryostat as an } \\
\text { option }\end{array}$ & 0 & Low \\
\hline Argon Purity & Cannot achieve required drift length & 3 & 1 & Med & Develop QA procedures & 0 & Low \\
\hline Argon Purity & $\begin{array}{l}\text { Argon purity immediately following filling is poor } \\
\text { requiring several volume recirulations before } \\
\text { operations can begin }\end{array}$ & 1 & 3 & Med & & 3 & Med \\
\hline Argon Purity & $\begin{array}{l}\text { Increasing the drift distance is a low impact } \\
\text { method of reducing the cost of LAr } 20 \text {, however } \\
\text { drift distances }>2.5 \mathrm{~m} \text { may not have been } \\
\text { demonstrated }\end{array}$ & 2 & 1 & Low & & 1 & Low \\
\hline Cryogenics & The cryostat develops a leak during operation. & 3 & 0 & Low & & 0 & Low \\
\hline Cryogenics & $\begin{array}{l}\text { Extrapolation from LNG experience/design to LAr } \\
\text { is invalid or not understood adequately }\end{array}$ & 3 & 1 & Med & $\begin{array}{l}\text { Engineering analysis will } \\
\text { mitigate this }\end{array}$ & 0 & Low \\
\hline Electronics & $\begin{array}{l}\text { "Hot electrons" at } 87 \mathrm{~K} \text { damage the cryogenic ASIC's } \\
\text { over the lifetime of the detector with a loss of } \\
>50 \% \text { of the detector channels }\end{array}$ & 3 & 1 & Med & $\begin{array}{l}\text { Perform stress test on } \sim 5 \% \text { of } \\
\text { Lar20 channel count }\end{array}$ & 0 & Low \\
\hline Management & $\begin{array}{l}\text { Suppliers are unable to deliver the needed } \\
\text { quantities of cryogens on the schedule needed for } \\
\text { filling }\end{array}$ & 2 & 1 & Low & & 1 & Low \\
\hline Management & $\begin{array}{l}\text { Critical components have a single supplier - } \\
\text { procurement \& operations }\end{array}$ & 2 & 0 & Low & $\begin{array}{l}\text { There is already an R\&D plan } \\
\text { in place }\end{array}$ & 1 & Low \\
\hline Management & $\begin{array}{l}\text { There are insufficient technical resources to } \\
\text { conduct the planned and proposed R\&D }\end{array}$ & 2 & 3 & High & Increase FNAL resources & 2 & Med \\
\hline Management & $\begin{array}{l}\text { There are insufficient scientific resources to } \\
\text { conduct the planned and proposed R\&D }\end{array}$ & 2 & 3 & High & Solicit additional collaborators & 2 & Med \\
\hline Physics R\&D & $\begin{array}{l}\text { There is no intermediate scale detector between } \\
\text { MicroBooNE and LAr } 20\end{array}$ & 1 & 1 & Low & & 1 & Low \\
\hline Physics R\&D & $\begin{array}{l}\text { No calibration exists to allow a determination of } \\
\text { EM and hadronic shower energy }\end{array}$ & 1 & 3 & Med & $\begin{array}{l}\text { Do a test beam calibration. } \\
\text { Use ArgoNeut? }\end{array}$ & 0 & Low \\
\hline Technical & $\begin{array}{l}\text { If drift distances }>2.5 \mathrm{~m} \text { are required, the required } \\
\mathrm{HV} \text { cannot be achieved }\end{array}$ & 3 & 1 & Med & $\begin{array}{l}\text { Operate a HV feedthrough } \\
\text { prototype }\end{array}$ & 0 & Low \\
\hline Technical & $\begin{array}{l}\text { Movement of long unconstrained wires creates } \\
\text { noise in the detector }\end{array}$ & 2 & 0 & Low & $\begin{array}{l}\text { SP: would like a small test on } \\
\text { long wires, in LN2 or dirty LAr }\end{array}$ & 0 & Low \\
\hline $\begin{array}{l}\text { Underground } \\
\text { issues }\end{array}$ & $\begin{array}{l}\text { The detector costs is significantly higher than } \\
\text { expected }\end{array}$ & 3 & 2 & High & $\begin{array}{l}\text { Track costing during } \\
\text { prototyping }\end{array}$ & 1 & Med \\
\hline $\begin{array}{l}\text { Underground } \\
\text { issues }\end{array}$ & $\begin{array}{l}\text { The cost of deployment at the deep underground } \\
\text { site is significantly higher than expected }\end{array}$ & 2 & 2 & Med & $\begin{array}{l}\text { Dry mockup to simulate } \\
\text { installation. } \sim 10 \% \text { size of } \\
\text { LAr } 20 . \text { Using Arup for UG } \\
\text { design \& estimating }\end{array}$ & 1 & Low \\
\hline $\begin{array}{l}\text { Underground } \\
\text { issues }\end{array}$ & $\begin{array}{l}\text { The ODH risk is not estimated correctly or the ODH } \\
\text { mitigation plan is not properly constructed }\end{array}$ & 3 & 1 & Med & $\begin{array}{l}\text { Perform an independent } \\
\text { review of FNAL ODH } \\
\text { methodology \& plan }\end{array}$ & 0 & Low \\
\hline
\end{tabular}

Figure 7: Risk matrix of post-MicroBooNE/LAPD risks and mitigation plan for these risks

\subsection{Risk Mitigation Action Items}

The final step in the analysis is to propose action items, or in our situation additional R\&D activities, that are necessary to reduce the likelihood of an event occurring. The consequence level is unchanged unless one redefines the scope of the study. The group determined a consensus opinion of the Mitigated Likelihood for each action item. The results of this process are shown in the risk matrix, Figure 7, and described below:

Here we briefly summarize the consensus view of the consequence and likelihood classification of each risk.

- Achievement of adequate lifetime requires evacuation. (MicroBooNE and/or LAPD were not successful).

The LAr20 reference design is based on the use of a membrane cryostat which cannot be evacuated. An evacuable modular cryostat will become the reference design if vacuum cleaning is required. The consequence level is difficult to estimate since a cost estimate for the modular 
cryostat option has not yet been developed.

We view the probability that evacuation will be required to be much less than $50 \%$ but greater than 1\%, based on a statistical physics argument. The Materials Test Stand results have shown that water is the primary contaminant affecting electron drift lifetime. Oxygen is another known contaminant however oxygen appears to be readily removed by the Oxysorb filters. These contaminants can be removed from the cryostat interior by creating an atmosphere in the cryostat with a very low partial pressure of water and oxygen; the gauge pressure inside the cryostat is unimportant.

- Cannot achieve required drift length.

The risk is that one cannot achieve the required electron lifetime in LAr20 even though LAPD and MicroBooNE demonstrated that this is possible. The most likely causes of such an event may be microscopic leaks through the LAr20 feedthroughs or a poor understanding of outgassing from cables or other components inside the cryostat.

The consequence and likelihood of this occurring are clearly difficult to quantify. Very minor leaks are much more likely than major leaks and may not affect detector performance significantly, but it is clear that careful qualification testing of components is required.

- Argon purity immediately following filling is poor, requiring several volume changes before operations can begin.

This risk was identified based on experience with ICARUS. We expect that a LAr TPC will generally have poor lifetime immediately after filling. The consequence level depends on the liquid argon recirculation rate and capacity of the purification system, which in turn, depend on the adequacy of the cryogenics design. We judge that with careful design, no more than a 3 month delay is likely.

- Increasing the drift distance is a low impact method of reducing the cost of LAr20, however drift distances $>2.5 \mathrm{~m}$ may not have been demonstrated.

The statement of this risk presumes that an adequate drift life lifetime has been demonstrated in MicroBooNE but unspecified problems arise when attempting to drift distances longer than $2.5 \mathrm{~m}$. For example, if the LAr20 design drift distance is set to $5 \mathrm{~m}$ to minimize cost and detector and signals from only the first $4 \mathrm{~m}$ of drift are useable, the consequence would be a $20 \%$ reduction in the fiducial mass. The likelihood of this event occurring depends on the results of the two $5 \mathrm{~m}$ drift tests in Europe. It is our opinion that a U.S. long drift test should be conducted if drift distance significantly longer than $2.5 \mathrm{~m}$ are proposed.

- The cryostat develops a leak during operation.

The consequence and likelihood of this risk clearly depend on the size of the leak. We relied on the results of a risk analysis of the GEOSTOCK underground LNG storage system in South Korea ["Risk Assessment of Membrane Type LNG Storage Tanks in Korea based on Fault Tree Analysis", Korean J. Chem. Eng. 22(1) 1-8 (2005)]. This paper reports that "we have only calculated the frequencies of occurrence in the gross mechanical failure which may lead to the release of a large amount of LNG or its vapor. The evaluated result shows this event frequency of $8.8 \times 10^{-6}$ /year which means this event may occur once in more than 110,000 years". The membrane cryostat used in GEOSTOCK was designed and constructed by Gaz Transport/Technigaz (GTT) in France, which appears to be the sole provider of this style of cryostat. 
- Extrapolation from LNG experience/design to LAr is invalid or not understood adequately.

The membrane cryostat is composed of stainless steel; a material of choice for LArTPCs. Liquid argon is $\sim 3 \mathrm{X}$ denser than LNG however, so the membrane weld method may need to be re-designed. We are confident that this difference can be adequately accounted for by engineering analysis.

- "Hot electrons" at 87K damage the cryogenic ASIC's over the lifetime of the detector with a loss of $>50 \%$ of the detector channels.

This is a well understood risk that is already being addressed by BNL, FNAL and Georgia Tech. The R\&D plan does not explicitly include testing of a fraction of the total electronics channel inventory ( $\sim 500 \mathrm{k}$ channels) however. The LAr20 electronics consultant, Marvin Johnson, recommends that a test be performed on $\sim 5 \%$ of the planned number of channels. This is a significant number of channels to test however it does not require a large volume cryostat. We are considering using the LAPD cryostat.

- Suppliers are unable to deliver the needed quantities of cryogens on the schedule needed for filling.

This appears to be an issue for European LAr TPCs. A procurement study done for the FLARE proposal two years ago showed that a $50 \mathrm{kt}$ detector would require $5 \%$ of the annual U.S. supply of liquid argon.

- Critical components have a single supplier - procurement $\&$ operations.

The only significant single supplier risk identified is the membrane cryostat. The manufacturer of membrane cryostats has been in operation of more than 30 years.

- There are insufficient technical resources to conduct the planned and proposed RED.

This is a common risk for new projects however the consequence for LAr20 is more severe. There is a limited time window available to demonstrate the viability of a LArTPC for long baseline neutrino oscillation physics. The current perception is that LArTPCs are a high risk and high reward technology. Adequate technical resources are required to do the R\&D to hopefully demonstrate that it is a low risk and high reward technology.

- There are insufficient scientific resources to conduct the planned and proposed RED.

The scientific community working on liquid argon for neutrino physics is relatively small. There is significant interest in the technology as demonstrated by the size of the MicroBooNE collaboration. Additional scientific resources are required to conduct simulations studies and to manage and conduct hardware R\&D in support of LAr20, however.

- There is no intermediate scale detector between MicroBooNE and LAr20.

This risk is our characterization of the opinion expressed by critics of the LAr R\&D plan that the jump from MicroBooNE to LAr20 is too large. The intent of this risk analysis is to reduce this statement to its component parts and to address these parts individually. The on-going and proposed R\&D plan does not include an intermediate detector that produces physics results. It is our judgment that MicroBooNE will demonstrate this capability and that it need not be duplicated in a somewhat larger detector. 
- No calibration exists to allow a determination of EM and hadronic shower energy.

ICARUS has published results on momentum resolution of MIP tracks using multiple scattering and range; however no LAr TPC has been calibrated in a test beam to allow measurement of electromagnetic and hadronic showers.

- If drift distances >2.5m are required, the required HV cannot be achieved.

The consequence of high voltage breakdown in a long drift TPC are severe, however the likelihood can be easily made low by prototyping.

- Movement of long unconstrained wires creates noise in the detector.

The ICARUS 3 ton prototype test experienced microphonic noise that was attributed to movement of the long anode wires. The cause of this wire movement was thought to due to convection currents or mechanical vibration. This noise has not been seen in any other LArTPC. The ICARUS T-600 detector includes wire restraints as a prophylactic measure. ANSYS calculations have demonstrated that convection currents in a reasonably insulated cryostat have insufficient driving force to cause this problem. Mechanical vibrations in an underground environment are negligible.

- The detector cost is significantly higher than expected.

This is a common risk for projects and has a high likelihood of occurrence. The consequence ranking is based on the assumption that the LAr20 cost estimate at CD-1 will be $\sim \$ 200 \mathrm{M}$ and the cost uncertainty will be $\sim 40 \%$.

- The cost of deployment at the deep underground site is significantly higher than expected.

This risk identifies the unique nature of constructing a massive underground detector. The consequence level is lower than the overall detector cost risk in recognition of the experience from other underground detectors (e.g. MINOS at Soudan).

- The Oxygen Deficiency Hazard (ODH) risk is not estimated correctly or the ODH mitigation plan is not properly constructed.

The consequence of this event is clearly very high; however it is our belief that the Fermilab cryogenic safety standard is adequate to control the hazard.

\subsection{Summary of the challenges and action items to mitigate risks}

\subsubsection{Argon Purity}

The effective operation of LArTPCs requires argon with contaminants less than 0.05 parts per billion (ppb) likely in very large, un-evacuable vessels. The questions we need to address and where to address them in the present program are as follows:

- What materials can be used which will not impact purity? The Materials Test stand at Fermilab is a facility expected to test for impact of materials that will go into MicroBooNE and LAr20.

- What purification system should be used with what arrangements of filters? The LAPD test will have a full purification system to exercise. Beyond this, MicroBooNE will continue to develop a full purification system now with a fully instrumented physics experiment. 
- What should the cleaning and fill procedure be? LAPD will provide the first test of this in an industrial built un-evacuable vessel. MicroBooNE will follow with a test using a fully instrumented physics experiment.

The membrane cryostat vessel for LAr20 must also be tested to ensure good purity can be achieved with this type of vessel. A small ( 20 ton) prototype will be constructed and tested to ensure good purity can be achieved and maintained.

\subsubsection{Electronics}

Development of cold electronics with multiplexing of signals inside the detector is crucial for LAr20. Cold electronics have the lowest noise levels available for this application. In addition, noise levels are minimized because the pre-amps are located at the end of the signal wires, minimizing noise due to capacitance of long wires. Finally, with their location at the signal wires, they provide flexibility in terms of detector geometry.

Cold pre-amps are already under development and test in the Bo electronics test stand at Fermilab. Fully integrated cold electronics are under development at Brookhaven and Fermilab and if ready in time, will be tested on some of the corner channels in the MicroBooNE experiment.

A test of $\sim 5 \%$ of the electronics to be used in LAr20 will be performed to mitigate the risks of channel failure.

\subsubsection{Active Detector System}

The active detector system is comprised of modules of TPCs and arrays of light collection devices. Many of the design parameters for the LAr20 TPC modules mimic those for MicroBooNE including the drift distance, wire pitch, wire plane spacing, etc. Much can be learned from MicroBooNE's TPC design, however, construction underground will drive the design specifics for LAr20. A full scale mechanical prototype of a LAr20 TPC module will be designed and constructed to study constructability, feasibility, integration with other systems and assembly. Assembly of the TPC prototype module will be done in a dry mock-up of part of LAr20. Provided there are no substantial design changes and no damage to the prototype, the TPC prototype can be re-used as one of the modules for LAr20.

This prototype will mitigate the technical, cost, and schedule risks associated with assembly and operation underground.

\subsubsection{Cryogenics}

The function of the cryogenic system for a liquid argon TPC detector is to provide and maintain a volume of liquid argon in a thermodynamic state that allows the TPC(s) to operate properly. The components of the system include a means of containing the argon, the cryostat, a means of delivery of liquid argon into the cryostat, a means of keeping the argon cold, a system of insulation to reduce the heat leak into the cryostat and a system to re-liquefy the argon boil-off and return it to the detector cryostat. While not necessarily cryogenic in nature, the cryogenic system will also provide a means to purge the cryostat of its atmosphere. A means of emptying the cryostat must also be provided as well as means to survive abnormal conditions such as power failure or non-delivery of nitrogen. The cryogenic system itself must not contribute any contamination that would reduce the electron drift lifetime in the liquid. The liquid in the detector cryostat must have a small temperature variation $(<1 \mathrm{~K})$ over its volume and the velocity of any currents in the liquid 
Integrated Plan/page 18

\begin{tabular}{|c|c|c|}
\hline Physics & Water & Argon \\
\hline \hline Long-Baseline Accelerator & 1000 mwe & $1-1000$ mwe \\
$\mathrm{p} \rightarrow K^{+} \nu$ & 3000 mwe & 3000 mwe \\
${ }^{8} B$ Solar $\nu$ & $<2400$ mwe & $<2400$ mwe \\
Supernova burst & 3500 mwe & 3500 mwe \\
Relic Supernova & 4300 mwe & $<2500$ mwe \\
Atmospheric $\nu$ & 2400 mwe & 2400 mwe \\
\hline
\end{tabular}

Table 2: Depths required for different physics goals for water Cherenkov and liquid argon detectors. (From the "Report on the Depth Requirements for a Massive Detector at DUSEL")

must also be small $\left(<0.1 \frac{\mathrm{m}}{\mathrm{s}}\right)$. And above all, the system above and below ground must be safe and satisfy all safety requirements.

This design, among other things, provides a base-line number for the heat-load. Systems to provide the cooling power required even for $100 \mathrm{kton}$ are available from several companies. Aspects of the cryogenic system for LAr20 will have been tested in devices which are expected to provide important input for the cryogenic system design. Operation of MicroBooNE will provide data on the purging of the cryostat, the effectiveness of a purely passive insulation scheme, and the process of re-liquefying and returning the Argon boil-off to the detector. The construction of MicroBooNE will also help qualify the selection of components such as valves and pumps. A validation of the model for predicting the behavior (motion and temperature distribution) of the liquid argon in the cryostat will, be provided by the Liquid Argon Purity Demonstration which is being equipped with a number of translatable temperature gauges precisely for this reason. The three major aspects of the LAr20 design which are new to us are: the construction in the DUSEL cavern of the membrane cryostat with its accompanying insulation, the (one-time) delivery of argon to this vessel almost one mile underground, and the provision of tens of tons of liquid nitrogen based cooling to a cryostat sited deep underground. These issues are outside our experience and we have turned to the companies which are the world-leaders in these areas to provide a conceptual design. Specifically, we have engaged Arup a world-renowned engineering consultancy. The Arup report will form the basis of any required $\mathrm{R} \& \mathrm{D}$.

\subsubsection{Underground Issues}

ArgoNeuT runs underground in the MINOS near hall, 100m below the surface, in an area classified with the lowest ODH risk (ODH 0). The safety review process for ArgoNeuT has helped us to identify issues related to underground operations, however, work beyond ArgoNeuT will be required as described below.

Two depth options are under consideration for LAr20 at DUSEL; the 300 (feet below ground) level and the 4850 level. The depth dependence of the physics capabilities of Water Cherenkov and liquid argon detectors was investigated in a 2008 study ("Report on the depth requirements for a massive detector at DUSEL"). The finding from this document, reproduced in Table 4.6.1, that is relevant for the integrated plan is that accelerator based neutrino oscillation physics can be conducted with a modest overburden (300 level) and potentially on the surface. Studies of non-accelerator based physics with a liquid argon detector can be performed at the DUSEL 4850 level.

The LBNE CD-0 mission need statement is expected to state the need for a long baseline accelerator based neutrino oscillation program. The task for the LAr20 project is to clearly delineate 
a) the cost and physics reach of LAr20 pursuant to the CD-0 mission statement from b) the incremental cost of including a non-accelerator based physics program that extends beyond the mission need statement. This distinction is important since DUSEL has drive-in access to the 300 level which would result in a significant cost savings over the 4850 level which is only accessible by shaft. The cost of mitigating the $\mathrm{ODH}$ risk is also expected to be significantly lower for a detector on the 300 level.

Cost Risk Mitigation: Experiences from other underground detectors (e.g. MINOS at Soudan) indicate that the installation labor cost may be $10 \%-30 \%$ higher than a surface detector. The labor premium clearly depends on the transverse size of the shaft and drifts, the travel time to the underground site and competition for shaft time by other parties. One component of the cost risk is mitigated by a fortuitous circumstance. The LBNE project has contracted with a consulting engineering firm (Arup) to perform the conceptual designs of the detector cryostat options and the cryogenics system. The design of the TPC that reside inside the cryostat, the electronics and the DAQ are outside the scope of the Arup contract. The conceptual design deliverables include a schedule and cost estimate for installation. Arup has more than 10 years of experience with the design of LNG storage and distribution systems as well as underground excavation and civil construction. Arup is also under contract with the DUSEL project to develop the design of the laboratory caverns at the 4850 level. We expect that Arup's past experience will result in a reliable cost estimate for a significant fraction of LAr20.

The LAr20 project team will develop the cost estimate for the TPC, electronics and DAQ. We propose to mitigate the attendant cost risk by constructing a mock-up of LAr20 sized to house one TPC module. The mock-up will be constructed of genuine cryostat components (including insulation) and a genuine TPC module. The mock-up will not include a cryogenics plant. The mock-up will be constructed in a facility (e.g. Wide Band Lab or D0) that will be altered to simulate the underground environment. Time and motion studies will be performed during construction. The mock-up will be constructed such that it could be converted into a functioning detector with the addition of a cryogenics plant, electronics and DAQ.

ODH Risk Mitigation: Reliable and safe operation of an underground detector containing 20 kton of cryogens is clearly of paramount importance. Such a large inventory poses a severe hazard that must be mitigated primarily by engineered controls. Similar hazards exist for accelerators and dark matter experiments but these facilities have a smaller inventory and more limited personnel exposure than is planned for LAr20. The DUSEL safety standards are still under development; however the LAr20 project has been informed that DUSEL will comply with OSHA standards. The LAr20 project office and the DUSEL project office have informally agreed that the LAr20 cryogenics design should be based on Fermilab safety standards, specifically by adhering to the Fermilab ODH and cryogenic safety standard.

This standard is well established and has been adopted by other institutions; nonetheless we must ensure 1) that the methodology has a sound basis for such a large inventory of cryogens in an underground environment, 2) that the application of the methodology to LAr20 is accurate and 3 ) that the review and approval mechanism of the mitigation plan is well defined. We will conduct a review of the ODH mitigation methodology and the proposed LAr20 mitigation plan by outside experts from industry (e.g. Factory Mutual), academia (e.g. UCSB Center for Risk Studies), and government (e.g. OSHA) and the mine safety arena. Representatives from DUSEL and SDSTA (South Dakota Science and Technology Authority) will be invited to participate in the review. 


\subsubsection{Physics R\&D}

No fully automated reconstruction package yet exists for LArTPC detectors. In addition, the only neutrino data yet collected with a LArTPC come from the exposure of a 50 liter prototype in the NOMAD beam at CERN in the 1990s, and now with the ArgoNeuT test. The Physics R\&D to be done with the existing program includes:

- Collect and analyze neutrino interaction data on Argon. This will be done first with ArgoNeuT which will collect $\sim 10 \mathrm{k}$ of neutrino interactions and later with MicroBooNE which will collect $>100 \mathrm{k}$ neutrino interactions and perform a suite of low energy cross section measurements. The neutrino interaction data from MicroBooNE will be used to

- Measure neutrino cross sections for LAr20 experiments including rare processes

- Measure signal like proton decay events and backgrounds to proton decay running on the surface

- Test cosmic ray background rejection while running on the surface

- Develop data compression schemes for continuous data taking

- Develop Supernova triggering capabilities

- Develop analysis tools for simulation and fully automated reconstruction for neutrino interactions and proton decay events. This will be done in the context of a software framework for the reconstruction and analysis of interactions in liquid argon, LArSoft, which is under active development. This framework is designed to provide universal algorithms for use by any LArTPC and currently supports the Bo and ArgoNeuT test stands as well as the MicroBooNE experiment. The modularity is accomplished by designing algorithms which are agnostic as to a particular LArTPC geometry so that the geometry for a specific LArTPC can be supplied at run time. The LArSoft developers group includes collaborators working on the test stands, as well as the MicroBooNE and LBNE experiments.

The scope of LArSoft is to perform all offline simulation, reconstruction, and analysis tasks. LArSoft can currently perform the following tasks: i) simulation of interactions in the detector, ii) tracking of particles through the detector, iii) simulation of readout electronics, iv) displaying of events. The simulation of interaction in the detector includes neutrino interactions using the GENIE package [http://www.genie-mc.org], cosmic ray interactions using the CRY package [http://nuclear.llnl.gov/simulation], and simulation of specific particles using LArSoft specific routines. The tracking of particles through the detector is accomplished using Geant4 [http://geant4.web.cern.ch/geant4]. The simulation of the readout electronics is different for each LArTPC, and the addition of different electronics for each new LArTPC is straight-forward.

The next challenge for the developers is to create reconstruction algorithms. A mature algorithm to identify individual signals on each wire of a LArTPC already exists. The next steps are to develop algorithms to group those signals into reconstructed tracks and showers. Discussions of various techniques and algorithms have started, and progress is expected over the next 4 to 6 months.

ArgoNeuT and MicroBooNE data will address nearly all the physics R\&D issues for LAr20. However, exposure of a LArTPC to a test beam will provide calibration data for electromagnetic and hadronic showers in addition to the present program. 


\section{Summary}

This document describes the challenges faced in scaling LArTPC detectors to 20ktons as required for the LBNE program. Over the last 3 months, the present and future LArTPC R\&D program in the US have been studied to develop a plan to address the challenges. Presented here is this plan which includes the existing program through MicroBooNE and steps beyond this to LAr20 at LBNE. The timeline with milestones for this Integrated Plan is shown in Table 4.3.4. The proposed R\&D activities are complete by the LAr20 final design milestone (CD3) in FY2014. 